\title{
Origine et destination du soin medical
}

\section{Philippe Barrier}

Philosophe, Docteur en sciences de l'éducation, Lauréat de l'Académie de Médecine,

Prix Pierre Simon « Ethique et Réflexion » 2014

barrierphilip@gmail.com

\section{Résumé}

En proposant une définition de l'usager intégrant tout utilisateur d'un service public à quelque titre que ce soit, c'est-à-dire aussi bien comme acteur professionnel que comme bénéficiaire, je propose une modélisation systémique et participative de la gestion de projet. Elle implique, dans le domaine du soin médical, l'idée d'un patient qui en est à la fois l'origine et la destination, et donc un acteur à part entière.

\begin{abstract}
Médical care: origin and destination - Defining the user as both professional and beneficiary, I propose a systemic and participative modelling of project management. This means, in the field of medical care, that the patient is both at the beginning and at the end of the process and that he is therefore an actor of care in his own right.
\end{abstract}

\section{Mots-clés}

Usager, Bénéficiaire, Auto-normativité, Conception systémique et participative

\section{Keywords}

User, Beneficiary, Self-normativity, Systemic and participative modeling

\section{Sommaire / Content}

Préambule

1. Des bénéficiaires acteurs, des acteurs bénéficiaires

2. Redéfinition de l'usager

3. Débouché philosophique de cette redéfinition

4. L'origine du soin médical

5. La destination du soin médical

Bibliographie 


\section{Ocula ${ }^{20}$}

La place de l'usager en design

Ph. Barrier | Origine et destination du soin medical I DOI: 10.12977/ocula2019-11

\section{Préambule}

Prenons comme postulat de départ l'assimilation courante de l'usager au patient dans le système de soin. Mon intervention concernant le champ de la philosophie du soin et de l'anthropologie médicale ne pouvait échapper à la question centrale de l'analyse critique de cette définition, en quelque sorte locale, du terme d'usager ; d'autant plus qu'elle m'interpelle directement. En effet, je me considère comme philosophe essentiellement parce que mon expérience vécue de la maladie m'a en quelque sorte imposé une approche phénoménologique de la pathologie, en même temps qu'une recherche en immersion sur la place du malade au sein du système de soin. Ma différence avec le chercheur, c'est qu'il n'y a rien de méthodologique dans mon positionnement de départ. La maladie est devenue pour moi un objet de pensée parce qu'elle est entrée dans mavie.

À ce titre, j'ai développé à la fois une conception du malade comme sujet humain confronté à une expérience singulière de la normativité de santé, et une analyse critique d'une relation patient-médecin fondée sur l'obéissance à une autorité détentrice d'un savoir considéré comme exclusif. Les deux problématiques sont évidemment liées. Ma définition ontologique du malade se double d'une définition sociologique du patient, parce que le malade se trouve d'emblée impliqué dans des rapports sociaux qui le conditionnent.

Le malade, pris en charge par un système de soin dont il est dépendant, devient un patient. Statut qui risque de lui faire perdre pas moins que son autonomie morale, car la relation de soin médicale tend à substituer à sa volonté propre une volonté de soin qui lui est extérieure ; elle tend à choisir le bien pour lui et à sa place, parfois même contre lui. La liberté quilui reste consiste à choisir entre le consentement ou le refus ; ce qui niela forme positive d'affirmation de salibertéqu'est le désir. Le désir de se soigner est exclus dans la représentation purement médicale du soin et du patient.

Mais le patient ne se trouve pas seulement réduit ontologiquement à ce positionnement de pure passivité, il l'est aussi administrativement, par sa réduction au statut d'usager, qui est en quelque sorte la version administrative de la passivité. Dans cette représentation, l'usager est le strict ou pur bénéficiaire d'un service qui lui est destiné. Ainsi le patient est le pur bénéficiaire du service hospitalier, l'élève est le pur bénéficiaire du système scolaire... On valorise ce statut et la bienfaisance du système, en soulignant depuis quelques années que le patient est au centre du système de soin, tout comme l'élève est au centre du système éducatif. Expressions qui ne signifient pas grand-chose, car on imagine mal le patient ou l'élève à la périphérie des services qui en ont la charge. 


\section{Ocula ${ }^{20}$}

La place de l'usager en design

Ph. Barrier I Origine et destination du soin medical I DOI: 10.12977/ocula2019-11

En fait, la notion de strict ou pur bénéficiaire a pour unique fonction de nier à l'usager toute légitimité à se considérer comme acteur du système dont il dépend et dont il est la finalité. Par exemple, pour le patient, à se considérer comme un acteur du soin. Il ne ferait que bénéficier du soin qu'on lui prodigue; c'est-à-dire le subir.

Or il me semble évident, d'une part, que le bénéficiaire est aussi acteur du système dont il bénéficie, d'autre part que les acteurs officiels du système (essentiellement les personnels qui y sont employés) en sont aussi bénéficiaires. Jevais tenter deprouverl'un etl'autre.

En contestant la représentation dominante de l'usager, je conteste du même coup celle du patient, et réciproquement. L'enracinement de cette double contestation se trouve dans une approche philosophique de la normativité entendue comme dynamique de régulation harmonieuse, directement issue de l'interprétation de la norme canguilhemienne telle que je la développe dans La blessure et la force (2010).

\section{Des bénéficiaires acteurs, des acteurs bénéficiaires}

Dans le cas du service hospitalier, son bénéficiaire, le patient à qui s'adresse le soin, est la cause finale de l'existence du service. C'est un rôle déterminant, assez vite oublié dans l'organisation d'un service, car comme toute organisation administrativement gérée, le servicetend à devenir à lui-même sa propre finalité. L'organisation se focalise facilement sur les problématiques de gestion du service et du personnel, en risquant de mettre au second plan sa finalité première, à savoir le soin, qui peut même s'y trouver subordonnée. Ainsi la logique des horaires tend à privilégier celle de la stricte gestion du personnel et non celledu soin, du patient, de ses proches. Il ne s'agit pas d'opposer une logique à l'autre, mais de prendre en égale considération l'une et l'autre, pour réguler et organiser le service. Ce rôle central dans la conception et l'organisation du service: penser àla satisfaction des besoins du patient, est retiré a priori au patient (qui en est pourtant le meilleur connaisseur). C'estuneautoritédécisionnelle, administrativeetgestionnaire, quipense à sa place, à partir de la représentation qu'elle en a.

On dénie donc au patient cette activité conceptuelle, car pour un mode d'organisation hiérarchique et exonomique (c'est-à-dire dont la norme de régulation est pensée comme extérieure) il est impensable que le bénéficiaire d'un service organisé puisse en être autre chose que son pur objet, c'est-à-dire un non-sujet. Même si, paradoxalement, on le sollicite après coup, dans le projet de réorganisation, en tant que sujet et acteur après l'avoir d'abord nié; d'où sa réticence et sa défiance.

Pourtant le patient se manifeste bien réellement comme sujet et acteur, en participant lui-même aux soins dont il bénéficie. D'abord parce qu'ilya consenti, c'est-à-dire qu'il a dit « non » à la possibilité de refus qui lui était offerte. Cette affirmation de la volonté qui refuse le refus, si elle est loin d'êtreletout dela liberté, en est néanmoins une sorted'expression positive minimale. Donc un acte effectif de choix, et un mode de partici- 


\section{Ocula ${ }^{20}$}

La place de l'usager en design

Ph. Barrier I Origine et destination du soin medical I DOI: 10.12977/ocula2019-11

pation actif à la prise de décision du soin, même si le patient n'en est pas l'initiateur. Il lui a fallu se battre de longues années pour obtenir ce droit au refus, accordé presque comme une concession ou une faveur, dont le patient n'ose d'ailleurs qu'assez rarement se servir.

De plus, très concrètement, le patient ne fait pas que subir passivement un soin, il y collabore (parfois très activement, comme dans le cas du test cardiologique del'effort), et de toute façon, ily réagit et en gère les effets etconséquences, leprolonge, leréfléchit. La pratique soignanteest en fait une interaction entre le soigné et le soignant, l'un et l'autre agissant, chacun dans le positionnement qui lui est propre, mais dont le cadre n'est malheureusement pas défini par les deux agents, mais par la seule autorité scientifique, technique, médicale et administrative, préalablement à l'activité.

Cequiapoureffet, maisaussi essentiellementpourbut,mesemble-t-il, degommerlapart activedupatient, voire, très concrètement, del'empêcher. Le choix de la position imposée aux femmes dans le dispositif d'accouchementen estunbel exemple: cetteposition n'est effectivement pratique et opératoire que pour l'accoucheur, et se montre non seulement inconfortable pour la parturiente, mais aussi inappropriée et antinaturelle pour l'activité d'expulsion qui est alors la sienne. On n'a sans doute pas cru nécessaire delui demander son avis.

De manière inversement symétrique aux patients, les différents personnels du service (médicaux, paramédicaux, administratifs, d'entretien, d'hôtellerie...), sont non seulement considérés comme acteurs exclusifs mais aussi comme exclusivement acteurs. Il se trouve qu'ils en sont cependant aussi des bénéficiaires, dans la mesure où ils en reçoivent un emploi, un salaire et un statut social et professionnel ; ce qui n'est pas un mince bénéfice. En fait, tout service public, bénéficie, sous une forme ou sous une autre, à tous ceux qui le pratiquent, que cette pratique soit reconnue ou non comme une activité.

Cette dichotomie artificielle entre usager (assimilé à un bénéficiaire) représenté comme purement passif, et agent représenté comme exclusivement et purement actif, recoupe, tout en la dissimulant, la distinction entre professionnels et non professionnels. La réduction du patient à un statut d'usager-bénéficiaire purement passif et celle de l'agent professionnel à celui de pur acteur a une fonction idéologique et pratique bien précise. Il s'agit d'exclure a priori l'usager-bénéficiaire, ici le patient, des prises de décisions concernant le service et son organisation. Mais pas seulement, car cette exclusion conforte le mode de décision et d'organisation hiérarchique, pyramidal, instauré et maintenu par les rapports entre employés hiérarchiquement et administrativement structurés. Ainsi le personnel, hors direction, se trouve donc également exclus des prises de décision.

\section{Redéfinition de l'usager}

Dans cette perspective critique, je propose donc qu'on puisse entendre par usager toute personne qui fait usage, d'une façon ou d'une autre, d'un 


\section{Ocula ${ }^{20}$}

La place de l'usager en design

Ph. Barrier I Origine et destination du soin medical I DOI: 10.12977/ocula2019-11

service public. Toute personne qui le fréquente, le pratique, s'y « coltine » en quelque sorte, c'est-à-dire qui se trouve confrontée à sa matérialité, à son espace, à son mode de circulation, d'organisation et de fonctionnement, et aussi aux représentations qu'il induit ou renforce. Ainsi, est usager d'un service hospitalier, par exemple, aussi bien le patient, ses proches ou accompagnants, que l'ensemble du personnel médical, paramédical, d'entretien, d'hôtellerie, de gestion, d'administration et de direction.

La représentation de l'usager ainsi proposée, supprimant la frontière entre bénéficiaires et agents professionnels, permet de sortir d'une conception pyramidale et pathogène de l'organisation, et de développer une représentation différente de la vie, au sens le plus fort, d'un service. Elle permet d'aborder réellement la problématique du design de service d'un point de vue systémique et participatif. Ce qui signifie pour moi, dans une approche à la fois spéculative et pragmatique, la possibilité de considérer une organisation humaine telle qu'un service public comme un écosystème au sens écologique du terme, c'est-à-dire comme un tout fonctionnant sur le principe de la symbiose, définie comme un ensemble d'interactions nécessaires mutuellement bénéfiques. Il s'agit là d'une tentative de modélisation, inévitablement simplificatrice.

Cequicaractériseunécosystème c'estla solidarité desélémentsquile constituent. Lebon fonctionnement du tout est dépendant du bon fonctionnement de chacun des éléments au sein du système. Autrement dit, le système fonctionne d'autant mieux qu'il fonctionne bien pour chacun dans son rapport à la finalité du système.

Ainsi, " solidaire » prend un sens vital qui redonne poids et signification au sens moral et politique du mot. La solidarité est une nécessité vitale du groupe humain, comme elle l'est de l'écosystème naturel. J'entends par là la reconnaissance et la prise en considération de l'interdépendance mutuelle des participants au système, en vue de son fonctionnement symbiotique. Dans cette perspective, la notion d'usager peut être entendue comme désignant l'ensemble exhaustifdes sujets en interactions réciproques, au sein d'un service - qu'on peut se risquer alors à qualifier de public.

Cela signifie que le projet d'organisation ou de réorganisation du service doit être le fait de tous ceux qui en font usage, sans exception. Cela implique l'égalepriseen considération des propositions de chacun, leur modélisation concrète, leur expérimentation, et enfin l'élaboration du meilleur compromis possible assurant la fluidité de fonctionnement du tout pour chacun, en rapport avec la finalité du service. L'organisation de cette confrontation est un lent, complexe et difficile travail d'observation, d'écoute et de proposition, en vue d'un possible dépassement, dans leur reconnaissance mutuelle, des représentations initiales dechacun.

Se trouve ainsi confortée une conception systémique et participative du projet de design de service, exemptée de la définition standard de l'usager, à la fois alibi et mauvaise conscience d'un système en dysfonctionnement. 


\section{Ocula ${ }^{20}$}

La place de l'usager en design

Ph. Barrier I Origine et destination du soin medical I DOI: 10.12977/ocula2019-11

\section{Débouché philosophique de cette redéfinition}

En prolongeant cette analyse, il me semble qu'on trouve au cœur de cette dynamique organisatrice, la potentialité normative spécifiquement humaine que j'ai appelé auto-normativité dans l'ensemble de mes travaux depuis La blessure et la force (2010). J'entends par là la spécificité de la dynamique régulatrice et équilibrante chez le vivant humain, qui n'est pas un être purement biologique, se satisfaisant de son homéostasie, mais aussi un être doué d'affectivité et d'intelligence symbolique, et donc aussi un être social et incomplet. Cette potentialité auto-normative lui permet à la fois de découvrir et gérer sa norme de santé, mais aussi son rapport aux autres dans ce qu'il a d'humainement vital, c'est-à-dire comme concourant non seulement à sa survie biologique, mais à sa vie en tant qu'être humain, c'est-à-dire en tant qu'être de relation.

La gestion systémique et participative du projet de design présente très clairement des caractères communs avec la dynamique auto-normative, en particulier le caractère inhérent de la règle (ou principe d'organisation) au phénomène à réguler (ou organiser). On peut y ajouter le fait que le principe d'organisation systémique et participatif se vérifie à son effet de fonctionnalité harmonieuse sur l'organisation, tout comme la norme se vérifie commenormeparl'effectivitédela régulation harmonieusequ'elleproduit, etqui n'est pas automatique.

\section{L'origine du soin médical}

Et c'est dans cette aptitude ou sensibilité normative universelle que s'origine le soin, comme l'explique Canguilhem, dans ce qu'il qualifie d'appel pathétique du malade (cf. 1966). Pathétique s'entendant ici dans le double sens de ce qui suscite une intense émotion, mais aussi de ce qui a trait au pathologique. En sachant que le pathologique n'est pas pour Canguilhem une catégorie objective et purement médicale, mais une catégorie subjective. C'est le malade qui définit ce qu'est le pathologique, parce que c'est lui qui l'éprouve.

Cette épreuve, cet éprouvé, est aussi une connaissance. Et c'est là que s'origine non seulement le soin, mais la médecine elle-même, comme le définit clairement cet admirable texte : "Nous pensons que la médecine existe comme art de la vie parce que le vivant humain qualifie lui-même comme pathologique, donc comme devant être écartés ou corrigés, certains états et comportements, appréhendés relativement à la polarité dynamique delavie, sousformedevaleurnégative. » (Canguilhem 1966 : p. 77)

C'est donc dans l'expérience du pathologique par « le vivant humain » (c'est-à-dire tout être humain) que s'origine la médecine, et donc le soin médical. Cette expérience n'est pas seulement un ressenti, mais une prise de conscience et une connaissance en même temps qu'une prise de décision et une action ; puisque l'homme est capable non seulement de qualifier ce ressenti comme pathologique, mais aussi, dans le même temps, d'en tirer les conséquences pratiques : le fuir, s'en protéger. 


\section{Ocula ${ }^{20}$}

La place de l'usager en design

Ph. Barrier I Origine et destination du soin medical I DOI: 10.12977/ocula2019-11

Cette qualification est d'emblée une prise de décision qu'on pourrait qualifier déjà de thérapeutique, puisqu'elle vise la mise à distance du pathologique. C'est grâce à cette initiale sensibilité répulsive au pathologique, que le malade convoque le médecin à son chevet et lui demande secours, lorsque ses propres forces normatives ne lui suffisent plus. La médecine n'a pas d'autre origine, si ce n'est le désir de répondre à cet appel, qui est la vocation première du médecin.

Mais c'est aussi sur cette potentialité auto-normative que le médecin va pouvoir s'appuyer pour réaliser la finalité éthique libératrice du soin. Car la médecine authentique va dans le même sens que cet élan normatif inhérent au vivant humain, et ne doit pas le contrarier. En effet: « le soin médical s'inscrit à l'intérieur du processus d'autorégulation propre à la nature » écrit Gadamer dans Philosophie de la santé (Gadamer 1992 : 48).

Il est donc de la responsabilité du médecin, avec ses connaissances médicales et cliniques, d'aider le patient à élucider les intuitions de cette conscience normative, pour qu'il parvienne à déterminer lui-même clairement le préférable ; qui est la norme, non dans la rigidité de sa définition abstraite ou statistique et prétendument universelle, mais dans la complexité et la finesse de sa réalité individuelle totalement singulière. Le médecin est donc le partenaire de ce travail normatif propre au patient.

\section{La destination du soin médical}

" L'art médical trouve son accomplissement dans le retranchement de soi-même et dans la restitution à autrui de sa liberté » écrit encore Gadamer, en insistant sur cette nécessité d'une sorte d'autosuppression de l'action médicale, qui marque son succès : "Toute action médicale trouve son accomplissement dans la suppression de soi. » (Gadamer 1992 : p. 48)

Cela semble aller de soi dans le cas de la guérison de la maladie aigue, mais en ce qui concerne la maladie chronique, c'est essentiellement en passant le relai au patient et en l'accompagnant dans ce soin qu'il se donne à lui-même. Tâche extrêmement complexe et noble, que les médecins ont encore du mal à intégrer à leur identité professionnelle, telle que leurs études et l'institution médicale l'a formatée.

La médecine doit apprendre l'humilité, c'est-à-dire apprendre à perdre son fantasme de toute puissance, tant envers son efficience thérapeutique qu'envers son pouvoir sur le sujet malade. L'institution de santé doit donc prendre conscience de l'origine et de la véritable destination du soin médical, afin de remplir correctement sa fonction d'aide irréductible à l'autonomie et la liberté du malade et d'organiser ses services en fonction de cettefinalité.

Ainsi le patient, loin de se réduire au statut d'usager passif et sans pouvoir décisionnel, pourra être authentiquement à la fois l'origine et la destination du soin médical, en tant que sujet libre, actif et responsable, coauteur etcoresponsable del'organisationdu service dont il est la finalité. 


\section{Ocula ${ }^{20}$}

La place de l'usager en design

Ph. Barrier I Origine et destination du soin medical I DOI: 10.12977/ocula2019-11

\section{Bibliographie}

Barrier, Philippe

2010 La blessure et la force, Paris, Puf.

Barrier, Philippe

2014 Le patient autonome, Paris, Puf.

Canguilhem, Georges

1966 Le normal et le pathologique, Paris, Puf.

Gadamer, Hans Georg

1992 Philosophie de la santé, Paris, Grasset-Mollat.

Leveque, Christian

2001 Ecologie, de l'écosystème à la biosphère, Paris, Dunod. 